\title{
INFLUENCIA DA QUALIDADE DA ÁGUA EM TROCADORES DE CALOR*
}

Rodrigo Martins Barbosa ${ }^{1}$

\section{Resumo}

A água é utilizada em processos industriais desde o começo da era industrial. Sua utilização é variada, desde como matéria prima, até como utilidades. Muito se discute a respeito da qualidade da água para descarte e seus possíveis danos ambientais, mas é importante mostrar que, além disso, existe um impacto financeiro ao trabalharmos com a má qualidade da água industrial. Este trabalho propõe demonstrar a influência da qualidade da água nos processos industriais, através da avaliação do resultado da instalação de um filtro de água na tubulação de entrada de um trocador de calor.

Palavras-chave: Trocador de calor; Filtro; Qualidade de água; Incrustação em trocadores de calor.

\section{WATER QUALITY INFLUENCE IN HEAT EXCHANGERS}

\begin{abstract}
Water is used in industrial processes since the beginning of the industrial age. Its use is diverse, goes from use as raw material, to use as utilities. There is a debate about water quality for disposal and its possible environmental damages, but it is important to show that in addition, there is also a financial impact by working with the poor quality of industrial water. This paper proposes to demonstrate the influence of water quality in industrial processes, by evaluation of the results of installing a water filter in the inlet of a heat exchanger.
\end{abstract}

Keywords: Heat exchanger; Strainer; Water quality; Heat exchanger fouling.

1 Engenheiro Químico, Engenheiro de Produtividade, Eficiência Operacional, Universidade Federal Fluminense, Niterói, Rio de Janeiro Brasil. 


\section{INTRODUÇÃO}

A qualidade de água tem impactos diretos à operação de processos industriais, tornando-se necessário o controle de qualidade da água, sendo feito através de monitoramento e tratamento. Os principais objetivos do tratamento são reduzir os impactos de:

- Formação de incrustações: é o acumulo de material nas paredes de equipamentos, prejudicando sua eficiência de operação.

- Processos Corrosivos: processo químico que consiste na destruição da estrutura original de um material metálico, através da retirada de elétrons do metal que transfere para o oxigênio.

- Controle Microbiológico: as condições físico-químicas da água podem gerar um ambiente adequado à formação de microrganismos.

Um dos principais usos da água é para o resfriamento de fluídos de processo, através de trocadores de calor. Será avaliado neste trabalho o impacto da formação de incrustação em um trocador de calor, e sua consequência no processo.

Para a análise da performance do trocador, serão utilizados os parâmetros de Approach, LMTD e o coeficiente global de transferência de calor (U).

\subsection{Approach}

Este parâmetro consiste na diferença entre temperatura de entrada de um dos fluidos e a saída do outro fluído no lado de maior relevância para o processo, ele é usado como uma medida simples de performance do trocador.

\subsection{LMTD}

$$
\text { Approach }=T_{\text {Saída do Gás }}-T_{\text {Entrada da Água }}
$$

O LMTD é a média logarítmica da diferença de temperatura entre o lado quente e o lado frio do trocador de calor, este leva em consideração a diferença de temperatura em todo o caminho do trocador.

Onde

$$
L M T D=\frac{\Delta T_{1}-\Delta T_{2}}{\ln \left(\frac{\Delta T_{1}}{\Delta T_{2}}\right)}
$$

$$
\begin{aligned}
& \Delta T_{1}=T_{\text {Saída do Gás }}-T_{\text {Entrada da Água }} \\
& \Delta T_{2}=T_{\text {Entrada do Gás }}-T_{\text {Saída da Água }}
\end{aligned}
$$

\subsection{Coeficiente Global de Transferência de Calor (U)}

Com este parâmetro é possível determinar o impacto da incrustação na performance do trocador de calor, este é dependente da geometria do trocador e de suas condições de projeto.

Onde:

$$
Q=U \times A \times L M T D
$$

$\mathrm{Q}=$ Calor Trocado no Sistema (W)

$\mathrm{U}=$ Coeficiente Global de Transferência de Calor $\left(\mathrm{w} / \mathrm{m}^{2} \mathrm{~K}\right)$

$\mathrm{A}=$ Área de Troca de Calor $\left(\mathrm{m}^{2}\right)$

LMTD = Diferença de Temperatura Média Logarítimica $(K)$ 
Para determinar o coeficiente global de transferência de calor, é considerado de forma simplificada o uso da equação de taxa de transferência de calor.

Onde:

$$
\boldsymbol{Q}=\boldsymbol{m} \times \boldsymbol{C p} \times \Delta \boldsymbol{T}
$$

$\mathrm{m}=$ Vazão mássica $(\mathrm{W})$

$\mathrm{Cp}=$ calor específico a pressão constante $(\mathrm{J} / \mathrm{kg} \mathrm{K})$

$\Delta \mathrm{T}=$ Diferença de Temperatura entre a entrada e saída do processo $(\mathrm{K})$

Se for considerada a primeira lei da termodinâmica, principio da conservação de energia, a energia transferida do fluído quente é igual à recebida do fluído frio, logo podemos considerar o calor gerado por um dos lados é o mesmo calor total do sistema.

$$
Q=U \times A \times L M T D=m \times C p \times \Delta T
$$

O coeficiente global fica então:

$$
U=\frac{m \times C p \times \Delta T}{A \times L M T D}
$$

A relação entre o coeficiente global de transferência de calor e incrustação é determinado pelo fator de incrustação que pode ser calculado através de:

$$
\frac{1}{U_{D}}=\frac{1}{U_{C}}+R_{D}
$$

$U_{C}=$ Coeficiente Global de Transferência de Calor "Limpo"

$U_{D}=$ Coeficiente Global de Transferência de Calor "Sujo"

$\mathrm{R}_{\mathrm{D}}=$ Fator de Incrustação

\subsection{Objetivo}

Com a melhora da performance do trocador de calor, conseguimos melhorar a temperatura de saída do gás, como há um separador de umidade e um compressor booster após o processo, é possível tirar mais água condensada do ar e reduzir a temperatura de sucção do booster, consequentemente reduzindo o consumo de energia elétrica.

\section{MATERIAIS E MÉTODOS}

O projeto consistiu na instalação de um segundo filtro do tipo chapéu de bruxa na entrada de água do trocador de calor, a fim de reduzir a quantidade de materiais a serem incrustados. No início da operação do compressor, já havia um filtro, mas devido ao fato de a qualidade de água ser bem baixa, era necessário abrir o caminho de "by-pass" com frequência para limpeza do filtro. 

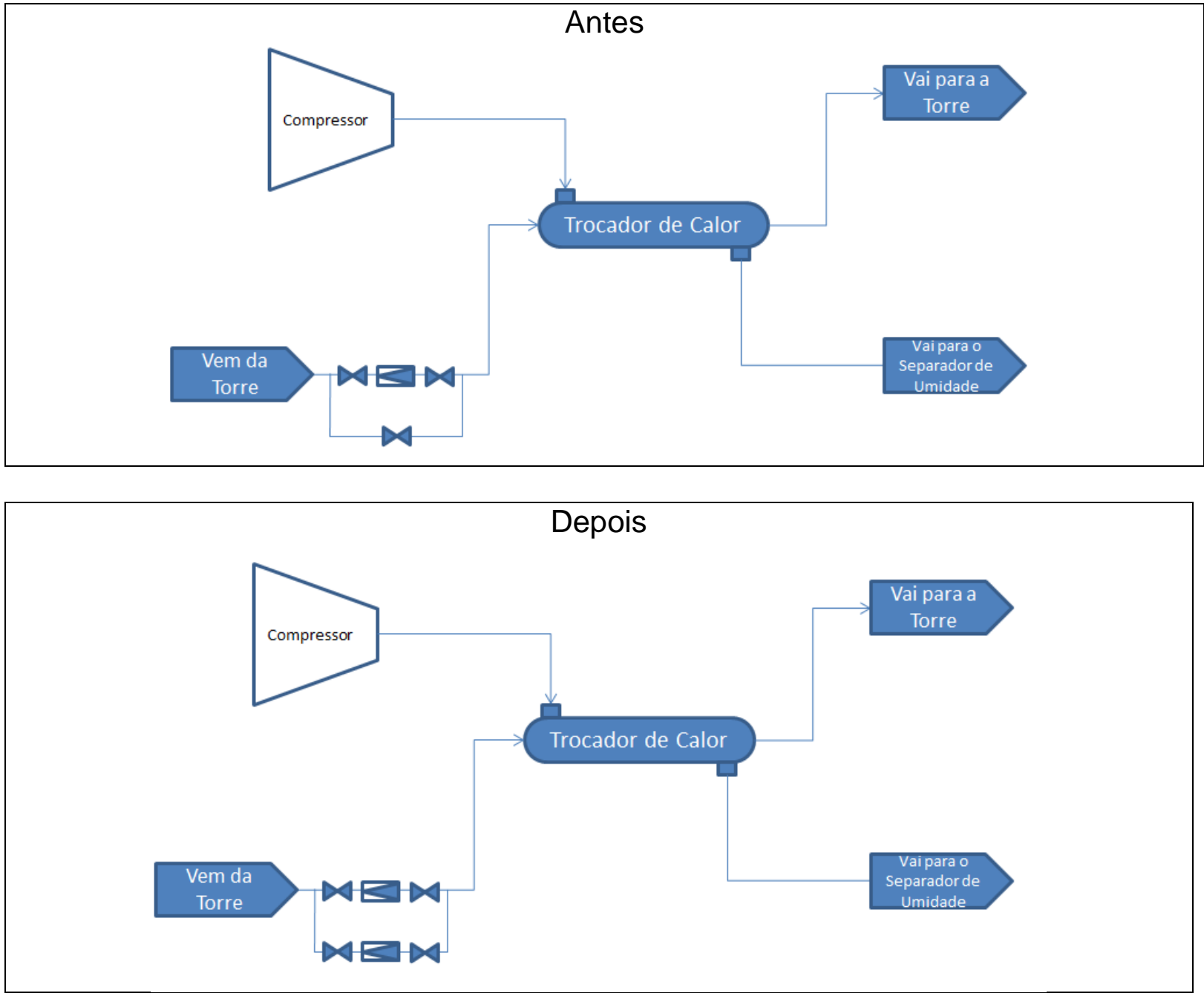

O trocador de calor estudado é do tipo casco tubo, este é o trocador de calor após a descarga do compressor de ar, suas características de projeto são:

\begin{tabular}{|c|c|c|}
\cline { 2 - 3 } \multicolumn{1}{c|}{} & Lado Casco & Lado Tubos \\
\hline Fluido & $\mathrm{Ar}$ & Água \\
\hline Temperatura de Entrada & $85^{\circ} \mathrm{C}$ & $33^{\circ} \mathrm{C}$ \\
\hline Temperatura de Saída & $41,3{ }^{\circ} \mathrm{C}$ & $4{ }^{\circ} \mathrm{C}$ \\
\hline Área de Troca Térmica & \multicolumn{2}{|c|}{$2.469 \mathrm{~m}^{2}$} \\
\hline Calor Trocado & \multicolumn{2}{|c|}{$5.597 .000 \mathrm{kcal} / \mathrm{h}$} \\
\hline
\end{tabular}

Dados Calculados:

LMTD - 20,8 K

$\mathrm{Uc}-126,85 \mathrm{~W} / \mathrm{m}^{2} \mathrm{~K}$

\section{RESULTADOS E DISCUSSÃO}

O compressor e o trocador de calor começaram a operar a partir de 25 de abril de 2012. A colocação do filtro foi realizada em março de 2015 , junto com uma manutenção de parada do compressor, pode ser demostrado os resultados expressivos através dos gráficos a seguir: 


\section{Approach}

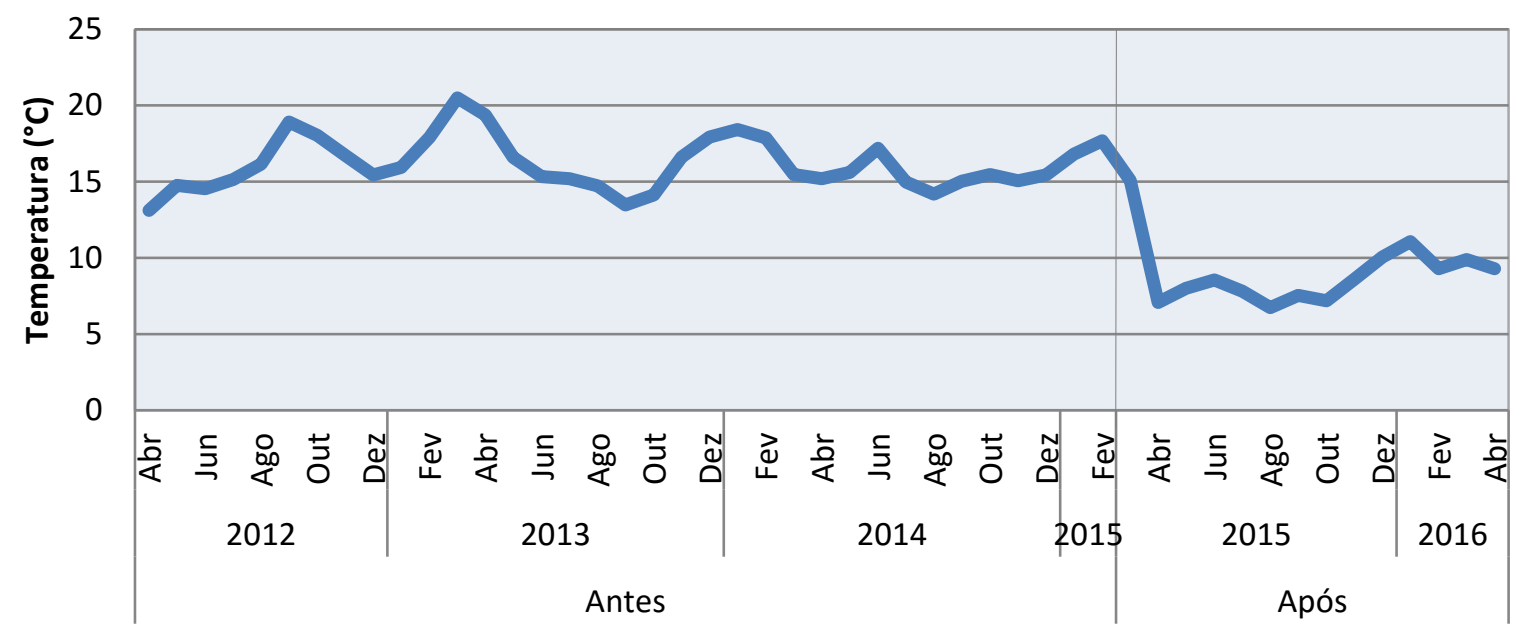

Figura 1. Gráfico de média mensal do approach do trocador desde 25 de abril de 2014 até 12 de abril de 2016.

Ao analisar apenas o approach, verificamos uma melhora significativa, uma redução de uma média de $16^{\circ} \mathrm{C}$ para $9^{\circ} \mathrm{C}$, ou seja, uma redução de $7^{\circ} \mathrm{C}$. Mas deve se levar em consideração que parte desta redução se deve ao fato de ter sido feita uma manutenção de tubos que foram perfurados ao longo da operação. Logo deve ser comparado ao início da operação quando podemos garantir que apenas a incrustação era o fator de queda de performance, sendo assim era $13^{\circ} \mathrm{C}$, o que representa uma queda de $1,4 \%$ na temperatura de saída do trocador, em termos absolutos (K).

Podemos comprovar a redução significativa da incrustação através do gráfico do fator de incrustação.

\section{Fator de Incrustação}

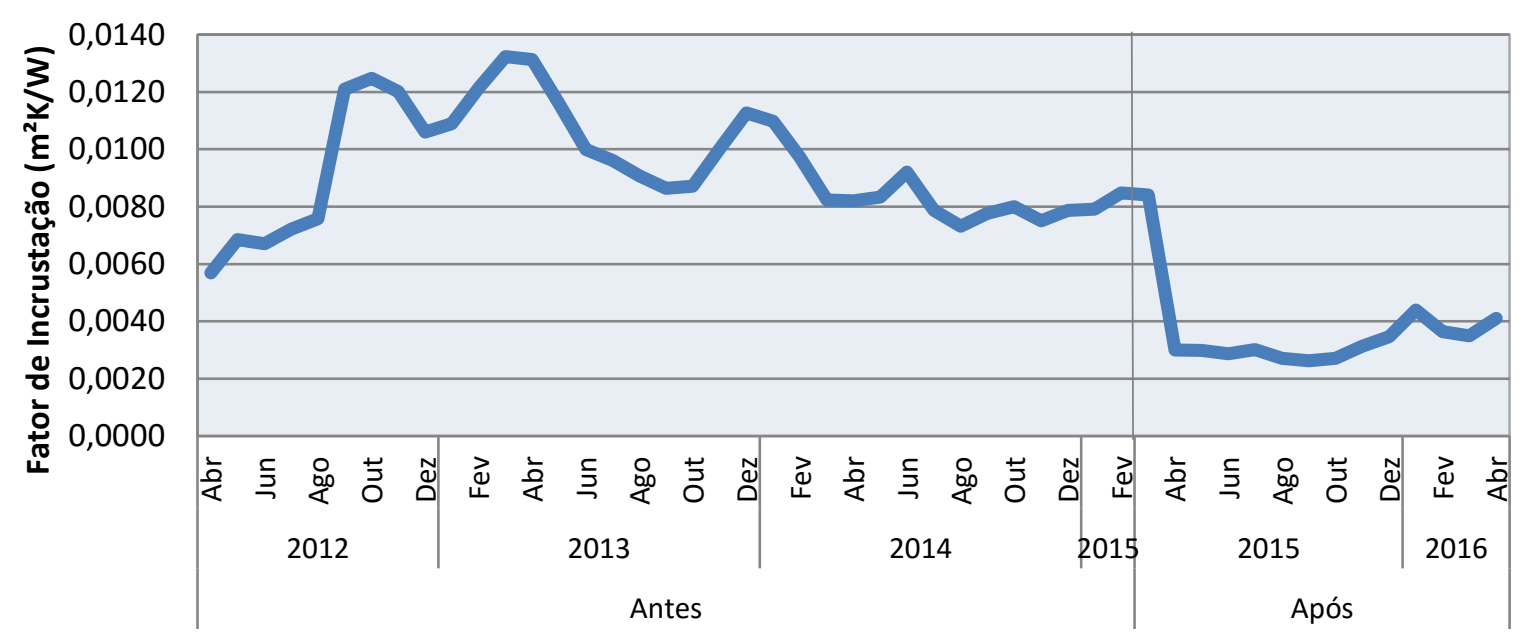

Figura 2. Gráfico de média mensal do fator de incrustação do trocador desde 25 de abril de 2014 até 12 de abril de 2016.

É possível observar, que se compararmos o mesmo período utilizado no approach, o fator de incrustação teve uma redução significativa de 0,0057 m²K/W para $0,0034 \mathrm{~m}^{2} \mathrm{~K} / \mathrm{W}$, representando uma queda de $65 \%$. 


\section{CONCLUSÃO}

O objetivo deste trabalho foi alcançado de maneira satisfatória, foi encontrada uma redução significativa na incrustação do sistema apenas com a instalação de um filtro simples, a má qualidade da água não é causadora só de impactos ambientais, mas também interfere na própria operação, quando se torna difícil ou demasiadamente custosa a implementação de sistemas de tratamentos mais avançados, torna-se necessária a implementação de soluções simples capazes de trazer um bom retorno.

Com a instalação do filtro pode se reduzir a quantidade de lavagens de um trocador de calor, por exemplo, reduzindo custos de mão de obra e material para uma tarefa rotineira.

O impacto mais significativo pode ser considerado na redução do consumo de energia elétrica, considerando a equação abaixo:

Onde:

$$
B H P=\frac{m \times C p \times T_{s}\left[\left(P d / P_{s}\right)^{\frac{k-1}{k}}-1\right]}{42.42 \times \eta_{a d}}
$$

BHP - "Brake Horse Power" adiabático (hp)

$\mathrm{Cp}=$ calor específico a pressão constante $\left(\mathrm{BTU} / \mathrm{lb} \mathrm{b}^{\circ} \mathrm{F}\right)$

$\mathrm{k}=\mathrm{Cp} / \mathrm{Cv}(1,403$ para o ar)

$\mathrm{m}=$ vazão mássica (lb/min)

$\mathrm{T}_{\mathrm{s}}=$ Temperatura de Sucção, $\left({ }^{\circ} \mathrm{R}\right)$

$\mathrm{P}_{\mathrm{d}}=$ Pressão de descarga (psia)

$P_{s}=$ Pressão de sucção (psia)

Observando a equação é possível notar que o BHP é diretamente proporcional à temperatura de sucção, se for considerado que todas as outras variáveis ficarão constantes, pode ser considerado então que uma redução de 1,4\% na temperatura de sucção do compressor representa uma redução na energia do compressor de aproximadamente mesma magnitude.

Devido aos compressores boosters consumirem somados cerca de $10 \mathrm{MWh}$, considerando os dados anteriores a redução seria de aproximadamente $140 \mathrm{~kW}$, este valor dentro do total se torna pouco perceptível, quaisquer outras variáveis do compressor que atuem interferem neste ganho, foi visualizada uma redução após fixarmos o valor de eficiência do compressor em $83 \%$ e a temperatura da água no trocador em $28^{\circ} \mathrm{C}$. 


\section{Consumo de Energia}

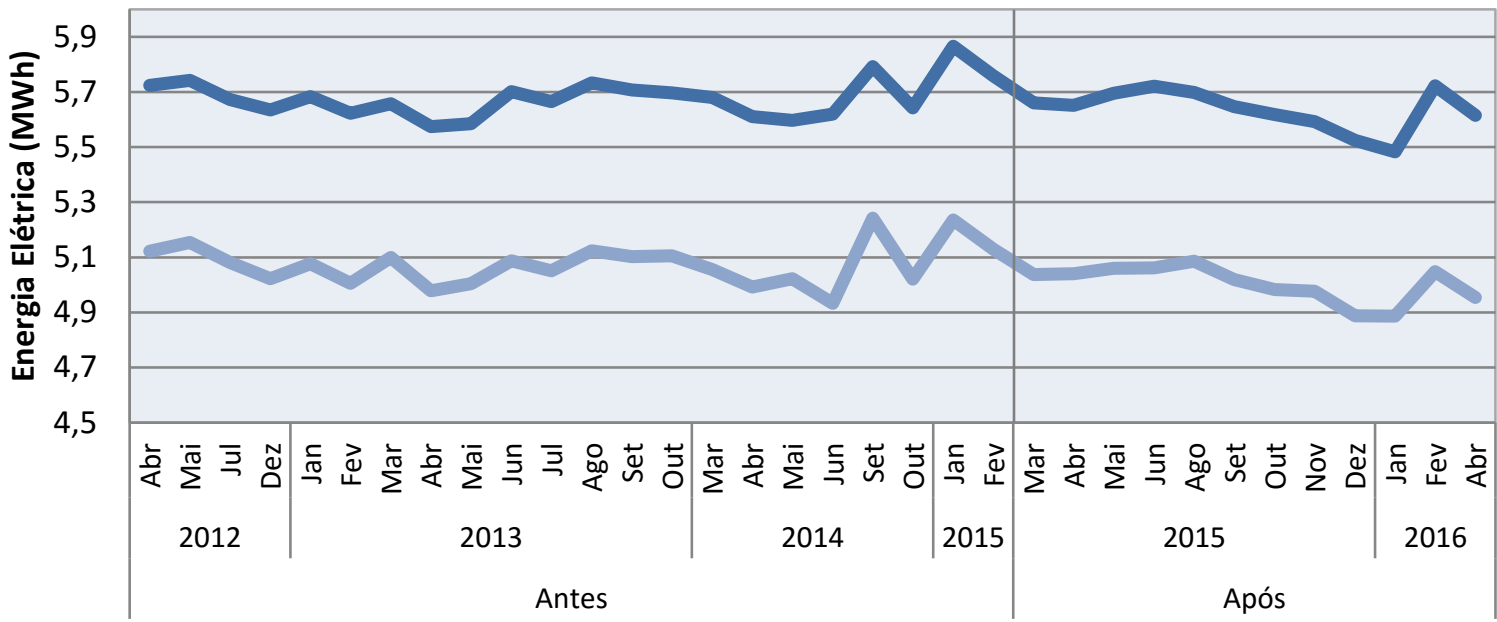

Consumo de Energia Elétrica Booster A

Consumo de Energia Elérica Booster B

Figura 3. Gráfico de média mensal do consumo de energia elétrica dos compressores boosters, com eficiência de $81 \%$ e temperatura de água no trocador de $28^{\circ} \mathrm{C}$, desde 25 de abril de 2014 até 12 de abril de 2016 .

Por se tratar de um sistema comum a todas as plantas da Praxair, é facilmente replicável.

\section{Agradecimentos}

Nossos agradecimentos vão para toda a equipe de operação e manutenção de nossa equipe de Volta Redonda, em especial para Edson Martins, supervisor de operação e Antônio Cabral, gerente da planta, também a Paulo Jasmim, engenheiro de manutenção e a Moises Gonzaga gerente de manutenção.

\section{REFERÊNCIAS}

1 SMITH, J. M.,; VAN NESS, H. C. Introduction to Chemical Engineerging Thermodynamics. 7 ed. Nova York: McGraw-Hill. 1959.

2 PERRY, R. H.,; Green, D. W. Perry's Chemical Engineers' Handbook. 8 ed Nova York: McGraw-Hill. 2008.

3 Kern, D. Q.,; Processos de Transmissão de Calor. Rio de Janeiro: Editora Guanabara Koogan S.A. 1980. 